\title{
Cantu syndrome and hypopituitarism: implications for endocrine monitoring
}

\author{
Nicholas J Theis ${ }^{1}$, Toby Calvert ${ }^{1}$, Peter Mclntyre², Stephen P Robertson ${ }^{2}$ and \\ Benjamin J Wheeler²
}

'Dunedin School of Medicine and 2Women's and Children's Health, Dunedin School of Medicine, University of Otago, Dunedin, New Zealand
Correspondence should be addressed to $B \mathrm{~J}$ Wheeler Email

ben.wheeler@otago.ac.nz

\begin{abstract}
Summary
Cantu syndrome, or hypertrichotic osteochondrodysplasia, is a rare, autosomal dominant genetically heterogeneous disorder. It is characterized by hypertrichosis, cardiac and skeletal anomalies and distinctive coarse facial features. We report a case where slowed growth velocity at 13 years led to identification of multiple pituitary hormone deficiencies. This adds to other reports of pituitary abnormalities in this condition and supports inclusion of endocrine monitoring in the clinical surveillance of patients with Cantu syndrome.
\end{abstract}

\section{Learning points:}

- Cantu syndrome is a rare genetic disorder caused by pathogenic variants in the $A B C C 9$ and KCN/8 genes, which result in gain of function of the SUR2 or Kir6.1 subunits of widely expressed $K_{\text {ATP }}$ channels.

- The main manifestations of the syndrome are varied, but most commonly include hypertrichosis, macrosomia, macrocephaly, coarse 'acromegaloid' facies, and a range of cardiac defects.

- Anterior pituitary dysfunction may be implicated in this disorder, and we propose that routine screening should be included in the clinical and biochemical surveillance of patients with Cantu syndrome.

\section{Background}

Cantu syndrome, first described in 1982, also known as hypertrichotic osteochondrodysplasia, is a heterogeneous syndrome characterized by hypertrichosis, cardiac and skeletal anomalies and distinctive coarse facial features (1). Although originally thought to be an autosomal recessive condition, inheritance was subsequently shown to be autosomal dominant $(1,2)$. To date, approximately 50 cases of Cantu syndrome and 12 of the closely related disorders acromegaloid facial features (AFF) and hypertrichosis with acromegaloid facial features (HAFF) have been reported. Among the 35 individuals with Cantu syndrome with reported testing, 28 had a pathogenetic variant in ABCC 9 gene and 2 in $K C N J 8$ (3). To date, those with a molecularly confirmed diagnosis of Cantu syndrome have been phenotypically similar to those with a purely clinical diagnosis, with data on AFF and HAFF being limited. Most cases represent de novo mutations in either ABCC9 or KCNJ8, which confer a 'gain of function' which affect the encoded proteins, the SUR2 and Kir6.1 subunits of $\mathrm{K}_{\mathrm{ATP}}$ channels. These genes are broadly expressed in most excitable tissues, including cardiac and skeletal muscle (4, 5). The mechanism by which these variants lead to the characteristic features of Cantu syndrome remains poorly understood, but similarities to clinical features seen with prolonged use of ATP-sensitive potassium channel agonists such as Diazoxide and Minoxidil (acromegaloid features and promotion of hair growth) support the role of $\mathrm{K}_{\mathrm{ATP}}$ channels in the pathogenesis of this condition (6).

Although there is substantial variable expressivity associated with this condition, all newborns have 
hypertrichosis, which usually persists, and many have macrosomia and macrocephaly. Cardiomegaly is frequent, and patent ductus arteriosus is present in about 50\% (3). The clinical phenotype among ten genetically confirmed cases from a number of countries was reported as 'coarse' facial features, flat nasal bridge, generalized hypertrichosis and at least one cardiac defect; seven also had recurrent infections (7). In terms of endocrine abnormalities, one case of Cantu syndrome with a KCNJ8 pathogenic variant had documented growth hormone deficiency, and familial non-functioning pituitary adenomata have been described in association with the syndrome in the context of a known $A B C C 9$ pathogenic variant $(2,8)$. We describe a case of confirmed Cantu syndrome with an ABCC9 pathogenic variant, presenting with progressive panhypopituitarism at the age of 13 years. We propose this has potential implications for the already complex surveillance recommended for patients with Cantu syndrome (3).

\section{Case presentation}

A 13-year-old boy with a clinically and molecularly confirmed diagnosis of Cantu syndrome was noted during routine follow-up to have a decreasing growth velocity. He had a height above the 85 th centile at 7 years 9 months (133.6 cm z-score 1.38), falling to 15 th centile $(154.0 \mathrm{~cm} \mathrm{z}$-score -1.05$)$ at 13 years 10 months. Growth velocity over the previous year was calculated at $2.9 \mathrm{~cm} /$ year (Fig. 1). His mid parental height was $180 \mathrm{~cm}$. He was experiencing mild fatigue, but was otherwise well, with no headaches or visual changes. There was no polyuria or polydipsia. On examination testicular volumes were
4 and $5 \mathrm{~mL}$ respectively, with some pubic hair growth consistent with Tanner stage 2; there was no axillary hair, deepening of voice, or other features of adrenarche. He had a dysmorphic appearance, with coarse facial features with pronounced hirsutism, consistent with descriptions of adults previously described with the disorder.

The patient's birth and developmental history was notable for preterm delivery at 36 weeks gestation, with marked hypertrichosis evident at birth. At 8 days of age, he returned to the pediatric assessment unit with clinical signs of heart failure and a 3/6 blowing systolic murmur. Echocardiography demonstrated coarctation of the aorta and a large patent ductus arteriosus. He subsequently underwent cardiac surgery to correct these abnormalities and required ongoing antihypertensives throughout infancy. When first seen in the Genetics Clinic at age 7 months, he had a dysmorphic facial appearance (pouting lips, flat midface, broad nasal bridge) and dense scalp hair, with a low posterior hairline and hypertrichosis covering the extensor surfaces of his limbs and back. These phenotypic features along with his background of congenital heart disease led to a clinical diagnosis of Cantu syndrome, which was subsequently confirmed in 2012 when genetic testing revealed a missense mutation in ABCC9, c. 3461G >A (p. Arg1154Gln) (4). Genetic studies at this time (including parental genotyping) confirmed this as a de novo mutation in the proband. The patient has since been presented in several papers describing the phenotypic and genetic characteristics of Cantu syndrome $(3,4,7)$.

At initial presentation at a chronological age of 13 years and 10 months, his bone age according to Greulich and Pyle was estimated at 12 years, 6 months (within

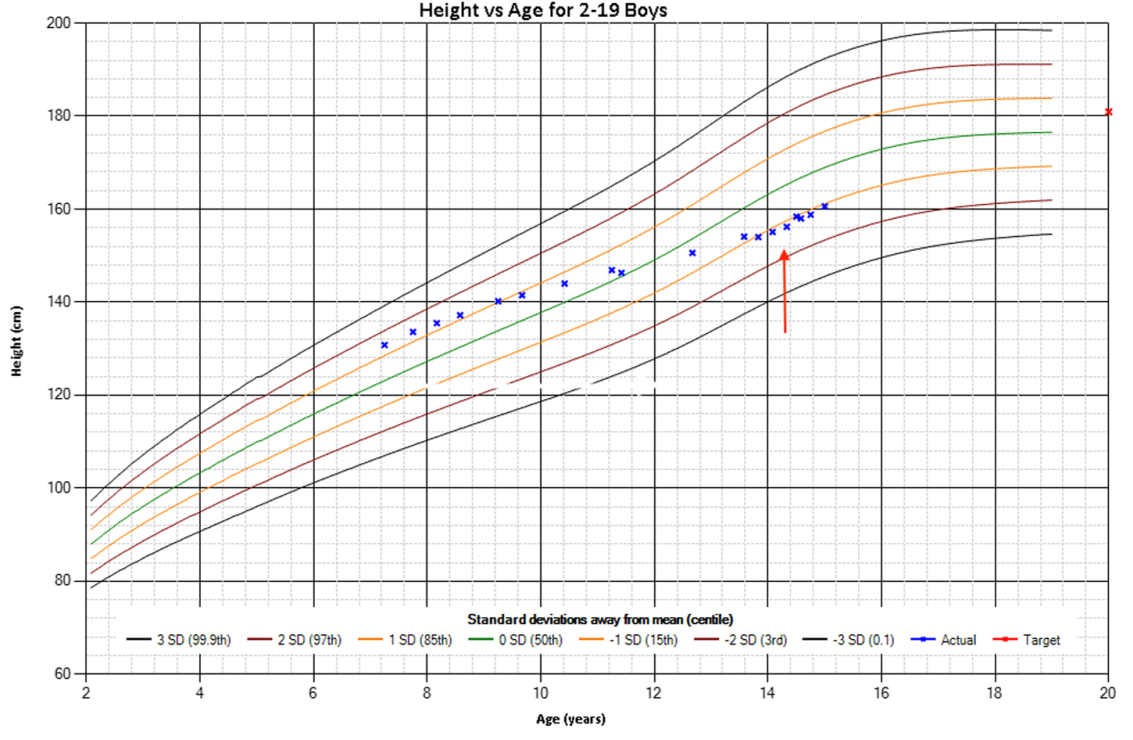

Figure 1

Electronic growth chart (Z-scores and graphs based on WHO child growth standards). Red arrow indicates point of growth hormone commencement. Red x - mid parental height estimate. 
2 standard deviations from the mean) (Fig. 2), IGF-1 was $51 \mu \mathrm{g} / \mathrm{L}$ (normal: 124-560) and growth hormone provocative testing (with estradiol priming) with arginine followed by clonidine confirmed partial growth hormone deficiency (peak growth hormone $2.32 \mu \mathrm{g} / \mathrm{L}$ (normal: $>5$ )). Testosterone was also pre-pubertal and low at $0.24 \mathrm{nmol} / \mathrm{L}$ (normal: 1.1-25.4), LH 2.0 IU/L (normal: <2.6), FSH 3.4 IU/L (normal: <6.6), and prolactin $599 \mathrm{mIU} / \mathrm{L}$ (normal: 50-350). TSH was $1.8 \mathrm{mIU} / \mathrm{L}$ (normal: 0.4-4.0) with a fT4 of $12.0 \mathrm{pmol} / \mathrm{L}$ (normal 10-24). Synacthen stimulation (250 $\mu \mathrm{g}$ ) demonstrated partial ACTH deficiency (peak cortisol $346 \mathrm{nmol} / \mathrm{L}$ (normal: >400)) but buserelin stimulation for potential hypogonadotropic hypogonadism was within normal limits (peak LH: $10.4 \mathrm{IU} / \mathrm{L}$ (normal $>10$ )). MRI revealed a small pituitary gland (height $4.0 \mathrm{~mm}$, normal $>6.0 \mathrm{~mm}$ ) but no intracranial mass and no evidence of posterior pituitary involvement (Fig. 3). Following these investigations (at 14 years and 3 months of age), he was

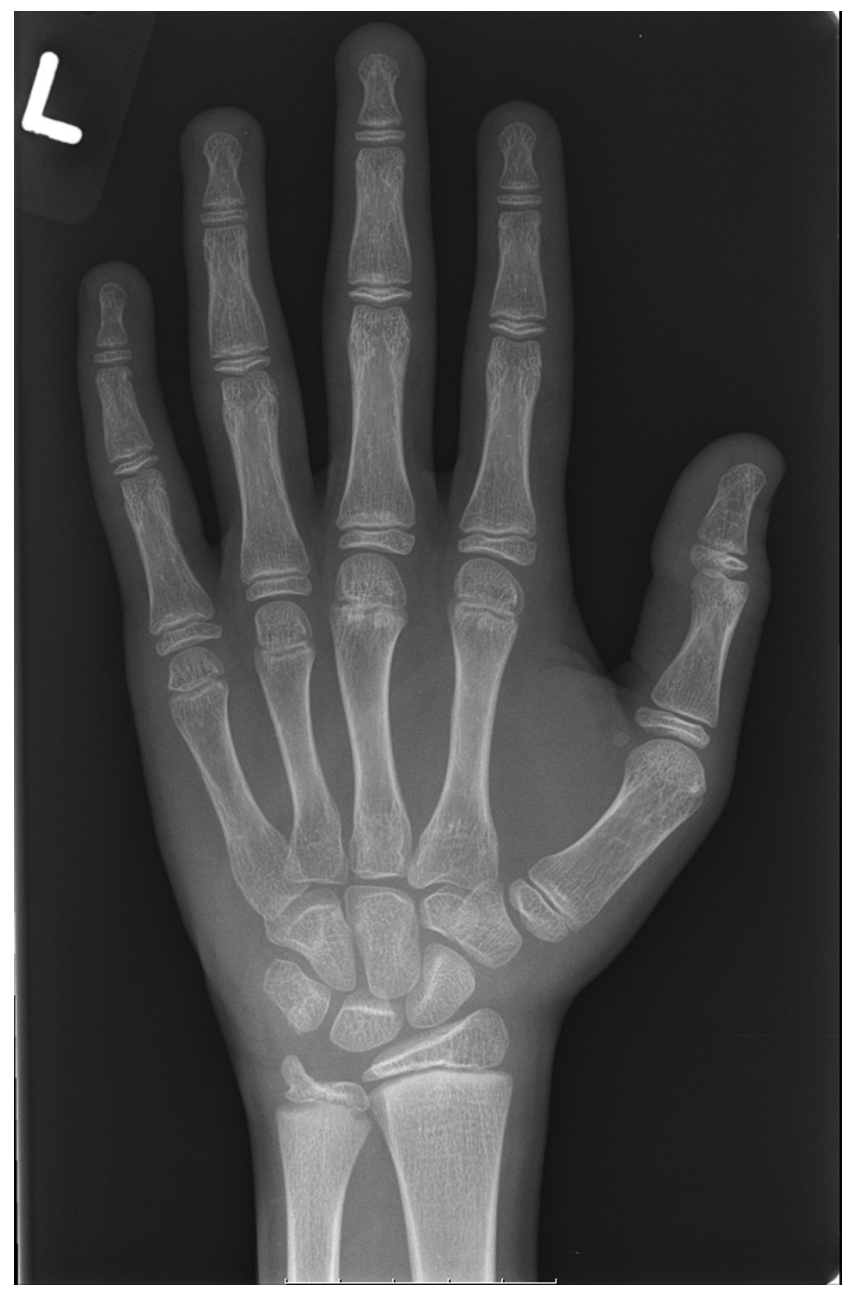

Figure 2

X-ray of left hand used for determination of bone age in the patient.
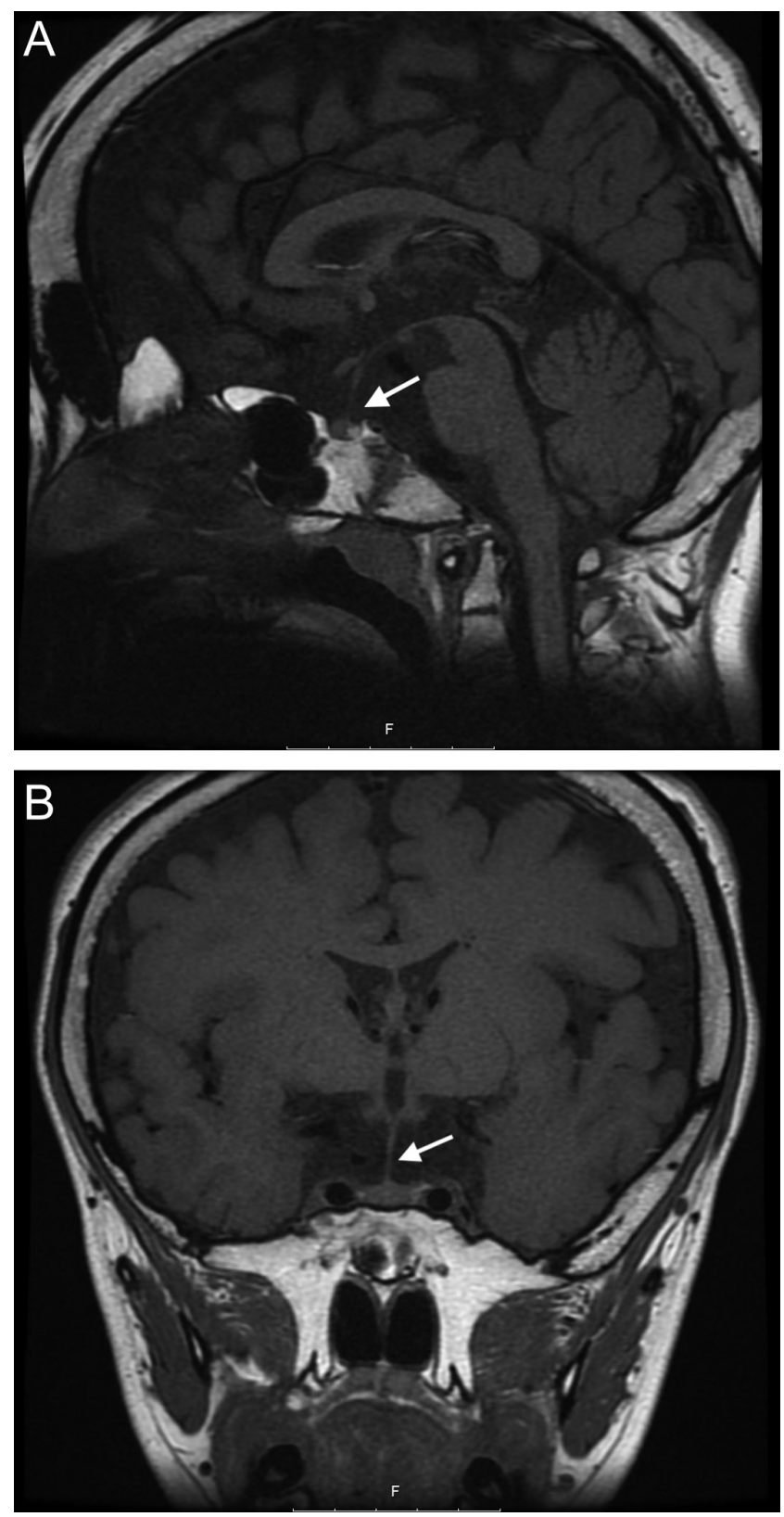

Figure 3

T1-weighted MRI sections demonstrating a small pituitary gland, with normal architecture (A: sagittal section, B: coronal section). White arrow indicates pituitary gland in pituitary fossa (Panel A) and in panel B indicates pituitary stalk.

commenced on $6.5 \mathrm{mg} / \mathrm{m}^{2} /$ week of growth hormone once daily and given an adrenal action plan for stress doses of hydrocortisone in the event of illness or injury.

\section{Outcome and follow-up}

In January 2019, due to ongoing malaise, Synacthen testing $(250 \mu \mathrm{g})$ was repeated (Table 1$)$. As there was 
Table 1 Anterior pituitary hormone levels.

\begin{tabular}{|c|c|c|c|c|}
\hline Parameters & $\begin{array}{l}\text { Reference } \\
\text { range }\end{array}$ & $\begin{array}{l}\text { June } \\
2018\end{array}$ & $\begin{array}{c}\text { January } \\
2019\end{array}$ & $\begin{array}{c}\text { March } \\
2019\end{array}$ \\
\hline Plasma IGF1, $\mu \mathrm{g} / \mathrm{L}$ & $124-560$ & 51 & 178 & \\
\hline Peak GH*,$\mu g / L$ & 5-10 & 2.32 & & \\
\hline Testosterone, nmol/L & $1.1-21.5$ & 0.24 & & 0.54 \\
\hline $\mathrm{LH}, \mathrm{IU} / \mathrm{L}$ & $<2.6$ & 2.0 & & \\
\hline FSH, IU/L & $<3.6$ & 3.4 & & \\
\hline Prolactin, mIU/L & $50-350$ & 599 & 450 & 514 \\
\hline $\begin{array}{l}\text { Peak cortisol**, } \\
\text { nmol/L }\end{array}$ & $>400$ & 346 & 114 & \\
\hline
\end{tabular}

*Peak GH, growth hormone following arginine and clonidine provocative testing; LH, luteinizing hormone; FSH, follicle-stimulating hormone; **Peak cortisol level following $250 \mu$ g Synacthen stimulation test.

progression of his adrenal insufficiency (peak cortisol of $114 \mathrm{nmol} / \mathrm{L})$, he was commenced on daily hydrocortisone ( $8 \mathrm{mg} / \mathrm{m}^{2} /$ day). On review 2 months later, he remained at Tanner stage 2 and his 0830 morning testosterone was $0.54 \mathrm{nmol} / \mathrm{L}$ (normal: 1.1-25.4), IGF-1 $269 \mu \mathrm{g} / \mathrm{L}$ (increased from $51 \mu \mathrm{g} / \mathrm{L}$ pre-treatment) with persistent mildly elevated prolactin (514 mIU/L) and normal thyroid function (fT4: $16.5 \mathrm{pmol} / \mathrm{L}$ ), prompting commencement of a pubertal induction dose of testosterone (40 mg daily, increased to $80 \mathrm{mg} 6$ months later; Table 1). Whether this will require conversion to full testosterone replacement therapy remains to be seen.

Eight months on from initially commencing growth hormone therapy (and following 6.5 months of hydrocortisone and low dose testosterone) height z-score has improved from -1.18 to -1.02 . In addition, his symptoms of fatigue have improved following the above hormonal interventions, now back to playing sport and attending school regularly (both of which had ceased prior to growth hormone and hydrocortisone). He has intermittently experienced mild peripheral oedema and arthralgia since commencing growth hormone therapy, but there have been no other changes to his previously described dysmorphic features.

\section{Discussion}

This case report, considered in conjunction with other similar case series and reports, suggests that pituitary abnormalities are a possible manifestation of Cantu syndrome. Cooper (5) describes a male with Cantu syndrome due to a de novo pathogenic variant in KCNJ8 who had growth hormone deficiency identified at 13 years of age; however, prior growth velocity was not recorded. Grange also reports 'growth hormone deficiency in a few individuals'; however, they do not provide any further clinical details (3). Other case reports include a patient with reported pituitary hyperplasia (7), a patient with an enlarged sella turcica (4), and a family with five affected family members, of which two (the proband and her maternal aunt) had non-functioning pituitary macroadenomata (8).

The mechanism for a specific endocrine effect in Cantu syndrome remains unknown; however, the expression profile and function of the affected $\mathrm{K}_{\mathrm{ATP}}$ channels may offer a possible explanation. The causative mutations linked to Cantu syndrome ( $A B C C 9$ and KCNJ8) encode the SUR2 and Kir6.1 subunits of $\mathrm{K}_{\text {ATP }}$ channels, structures which regulate an important step in the coupling of cell metabolism to electrical excitability, as well as playing a role in metabolic pathways and the regulation of hormone secretion. These mutations result in a 'gain-of-function' effect which reduces ATP-mediated inhibition of $\mathrm{K}_{\text {ATP }}$ channels, thereby effectively increasing $\mathrm{K}_{\mathrm{ATP}}$ channel activity (4). Interestingly, $\mathrm{K}_{\mathrm{ATP}}$ channel activity comprising Kir6.2 and SUR1 (closely related subunits of the same channel affected in Cantu syndrome) are found in glucosesensing neurons within the hypothalamus controlling growth hormone release in response to hypoglycemia. A transient increase in opening of these $\mathrm{K}_{\text {ATP }}$ channels is required to initiate a physiologic counter-regulatory response (CRR) to hypoglycemia, which includes the release of cortisol and growth hormone (9). Therefore, it is plausible that persistently elevated $\mathrm{K}_{\mathrm{ATP}}$ channel activity in Cantu syndrome could adversely impact growth hormone release. Our patient also suffered from partial adrenal insufficiency, which further supports the notion of a link between Cantu syndrome and dysregulation of central glucose-responsive hormone release.

Similar links to the hypothalamic-pituitary-gonadal (HPG) axis could provide a mechanistic explanation for our patient's low pre-pubertal testosterone levels. Several lines of evidence, including clinical and in vitro studies, also demonstrate an important role for inwardly rectifying potassium channel $\left(\mathrm{K}_{\mathrm{IR}}\right)$ activity in regulation of gonadotropin-releasing hormone $(\mathrm{GnRH})$ secretion. These effects are mediated by the Kir3.1 and Kir3.4 channels, both related to the Kir6.1 subunit which is mutated in Cantu syndrome. Secretion of GnRH by hypothalamic neurons has been shown to rely on potassium-induced membrane depolarization, and persistently open $\mathrm{K}_{\mathrm{IR}}$ channels secondary to a gain-of-function mutation would negate this process and thereby potentially impact GnRH secretion (10). 
n the context of recent characterization of the causative mutations in Cantu syndrome, along with the known physiologic impacts of these mutations on potassium channel $\left(\mathrm{K}_{\mathrm{IR}}\right)$ activity, there is significant research interest in possible therapeutic targets which may offer clinical benefit in these patients. Of note, sulfonylurea drugs which act as potent inhibitors of $\mathrm{K}_{\text {ATP }}$ channel activity, show promise. If recent suggestions for therapy for Cantu syndrome prove efficacious, it might also impact on this rare manifestation of the disorder, if indeed these endocrine defects prove to have a functional basis relating to the $\mathrm{K}_{\text {IR }}$ potassium channel defect (6).

Cantu syndrome is a rare genetic syndrome which arises due to missense mutations in $A B C C 9$ or KCNJ8, resulting in a gain-of-function of the widely expressed $\mathrm{K}_{\mathrm{ATP}}$ channel. It is associated with a wide array of phenotypic characteristics, the most common being hypertrichosis, cardiac and skeletal anomalies, and distinctive coarse facial features described as 'acromegaloid' in appearance. We describe a patient with the syndrome with evolving multiple anterior pituitary hormone deficiencies, initially identified through slowed growth velocity at age 13 years. We propose a mechanistic link between $\mathrm{K}_{\text {ATP }}$ channel mutations and pituitary function which may explain pituitary dysfunction in our patient. The accumulating number of reports of pituitary pathology in Cantu syndrome supports a possible pituitary association as part of the syndrome. Surveillance of growth velocity and pituitary hormone screening are simple additions to current surveillance recommendations (cardiac status and behavioral and cognitive development) which would allow early recognition of significant treatable endocrine disease in patients with Cantu syndrome.

\section{Declaration of interest}

The authors declare that there is no conflict of interest that could be perceived as prejudicing the impartiality of this case report.

\section{Funding}

This research did not receive any specific grant from any funding agency in the public, commercial or not-for-profit sector.

\section{Patient consent}

Written informed consent has been obtained for publication of the submitted article and accompanying images.

\section{Author contribution statement}

$\mathrm{N} J \mathrm{~T}$ and $\mathrm{T} C$ were involved in preparation of initial draft and wrote the manuscript. B J W supervised N J T and T C in case report preparation and is the patient's pediatric endocrinologist and supervised all the reported endocrine testing. S P R is the patient's clinical geneticist and was involved in identifying and characterizing the patient's mutation. P M was involved in case report development and supervision and manuscript editing. All authors were involved in revision and approval of the final manuscript.

\section{References}

1 Cantu JM, Garcia-Cruz D, Sanchez-Corona J, Hernandez A \& Nazara Z. A distinct osteochondrodysplasia with hypertrichosis individualization of a probable autosomal recessive entity. Human Genetics 198260 36-41. (https://doi.org/10.1007/bf00281261)

2 Grange D, Nichols C \& Singh G. Cantu syndrome and related disorders. In GeneReviews ® [Internet]. Eds MP Adam, HH Ardinger \& RAAlEd Pagon. Seattle: University of Washington, 1993-2019. (available at: https://www.ncbi.nlm.nih.gov/books/NBK246980/)

3 Robertson SP, Kirk E, Bernier F, Brereton J, Turner A \& Bankier A. Congenital hypertrichosis, osteochondrodysplasia, and cardiomegaly: Cantu syndrome. American Journal of Medical Genetics 199985 395-402. (https://doi.org/10.1002/(SICI)10968628(19990806)85:4<395::AID-AJMG17>3.0.CO;2-I)

4 van Bon BWM, Gilissen C, Grange DK, Hennekam RCM, Kayserili H, Engels H, Reutter H, Ostergaard JR, Morava E, Tsiakas K, et al. Cantú syndrome is caused by mutations in ABCC9. American Journal of Human Genetics 201290 1094-1101. (https://doi.org/10.1016/j. ajhg.2012.04.014)

5 Cooper PE, Reutter H, Woelfle J, Engels H, Grange DK, van Haaften G, van Bon BW, Hoischen A \& Nichols CG. Cantú syndrome resulting from activating mutation in the KCNJ8 gene. Human Mutation 201435 809-813. (https://doi.org/10.1002/humu.22555)

6 Ma A, Gurnasinghani S, Kirk EP, McClenaghan C, Singh GK, Grange DK, Pandit C, Zhu Y, Roscioli T, Elakis G, et al. Glibenclamide treatment in a Cantú syndrome patient with a pathogenic ABCC9 gain-of-function variant: initial experience. American Journal of Medical Genetics: Part A 2019179 1585-1590. (https://doi org/10.1002/ajmg.a.61200)

7 Scurr I, Wilson L, Lees M, Robertson S, Kirk E, Turner A, Morton J, Kidd A, Shashi V, Stanley C, et al. Cantú syndrome: report of nine new cases and expansion of the clinical phenotype. American Journal of Medical Genetics: Part A 2011 155A 508-518. (https://doi. org/10.1002/ajmg.a.33885)

8 Marques P, Spencer R, Morrison PJ, Carr IM, Dang MN, Bonthron DT, Hunter S \& Korbonits M. Cantú syndrome with coexisting familial pituitary adenoma. Endocrine 201859 677-684. (https://doi. org/10.1007/s12020-017-1497-9)

9 Miki T, Liss B, Minami K, Shiuchi T, Saraya A, Kashima Y, Horiuchi M, Ashcroft F, Minokoshi Y, Roeper J, et al. ATP-sensitive K+ channels in the hypothalamus are essential for the maintenance of glucose homeostasis. Nature Neuroscience 20014 507-512. (https:// doi.org/10.1038/87455)

$10 \mathrm{Hu}$ L, Wada K, Mores N, Krsmanovic LZ \& Catt KJ. Essential role of $G$ protein-gated inwardly rectifying potassium channels in gonadotropin-induced regulation of GnRH neuronal firing and pulsatile neurosecretion. Journal of Biological Chemistry 2006281 25231-25240. (https://doi.org/10.1074/jbc.M603768200)

Received in final form 18 October 2019

Accepted 23 October 2019 